\title{
Utilización de Servicios de Salud y Perfiles Epidemiológicos como Parámetros de Adecuación del Plan Obligatorio de Salud en Colombia
}

\author{
Luis Alejandro Gómez ${ }^{1}$, Hilda Constanza Tovar ${ }^{2}$ y Carlos A. Agudelo $C^{3}$ \\ ${ }^{1}$ Odontólogo. M. Sc. Salud Pública. Hospital del Sur-Empresa Social de Estado. \\ E-mail: alegomezbar@yahoo.com \\ ${ }^{2}$ Médica. M. Sc. Salud Pública. Médicos Asociados, Bogotá. E-mail: htovarm@hotmail.com \\ ${ }^{3}$ Médico. M. Sc. Salud Pública. M. Sc. Ciencias. Instituto de Salud Pública, Facultad de Medicina, \\ Universidad Nacional de Colombia. E-mail: caagudeloc@unal.edu.co
}

\begin{abstract}
Objetivos Evaluar la utilización de los servicios de salud y los perfiles epidemiológicos, como parámetros de ajuste de los contenidos del Plan Obligatorio de Salud-POS.

Metodología Se realizó un estudio evaluativo de los contenidos del POS con respecto a la utilización de servicios y los perfiles epidemiológicos regionales, en el marco de la problemática actual del Sistema General de Seguridad Social en Salud. La utilización de los servicios se estimó por medio de la Encuesta sobre Equidad en la Gestión Fiscal de 1994, la Encuesta Nacional de Calidad de Vida de 1997 y la Encuesta Nacional de Hogares de 2000. Los perfiles epidemiológicos se obtuvieron de los datos de mortalidad del DANE del año 2000.

Resultados La afiliación al sistema de salud ha logrado llegar sólo a un $56 \%$ del total de la población. Sin embargo, el aumento del gasto en salud ha sido constante entre 1993 y el año 2000, pasando de un $7,2 \%$ del PIB a cerca de un $10 \%$. Al tiempo se observan diferencias en la mortalidad por regiones. Se encontró mayor utilización de servicios a mayor edad y a mayor nivel de gasto de las personas. Las mujeres son las que más utilizan servicios de salud, especialmente las que residen en zonas urbanas y en el quintil de gasto más alto. La menor utilización es la de los hombres residentes en la zona rural, del primer quintil de gasto.

Conclusiones Organizando el POS con una estructura modular es posible ajustarlo por medio de los parámetros de utilización de los servicios y los perfiles epidemiológicos, de tal manera que contribuya a mejorar los problemas de oportunidad y pertinencia de los servicios, así como acrecentar la afiliación al sistema.
\end{abstract}

Palabras Claves: Sistema de salud, servicios de salud, epidemiología, seguro de salud (fuente: DeCS, BIREME).

\section{ABSTRACT}




\section{Use of health services and epidemiological profiles as parameters for adjusting the Compulsory Health Plan in Colombia}

Objective To evaluate the use of health services and the epidemiological profiles as parameters for adjusting the contents of the Compulsory Health Plan-POS.

Methods An evaluative study of the contents of the Compulsory Health Plan-POS was performed regarding the use of health services and the regional epidemiological profiles, within the framework of the current problems of the General System of Health Social Security. The 1994 Survey on Equity of the Fiscal Expenditure, the 1997 National Survey on Quality of Life, and the 2000 National Household Survey estimated the use of services. The epidemiological profiles were obtained from the 2000 mortality data of the National Administrative Statistics Department-DANE.

Results Affiliation to the health system has only reached $56 \%$ of the total population. However, health expenditure has increased constantly between 1993 and 2000, from 7,2 \% to nearly $10 \%$ of the GNP. Greater use of health services was found among the elderly population, and people with greater levels of spending. Women, particularly those residing in urban areas and on the higher quintile of spending, are the people who most use the health services. The people who use health services the least are men in urban areas in the lower quintile of spending.

Conclusions Organizing the Compulsory Health Plan with a modular structure makes it possible to adjust it according to the parameters of the use of health services and epidemiological profiles, in such a way that it contributes to the opportunity and pertinence of services, as well as to increase the affiliation to the system.

Key Words: Healthcare system, health service, epidemiology, insurance (source: MeSH, NLM)

$\mathrm{E}$ l Sistema General de Seguridad Social en Salud-SGSSS fue creado mediante la Ley 100 de 1993, con el fin de reemplazar el antiguo Sistema Nacional de Salud-SNS que funcionaba desde 1975, basado en un esquema centralizado en el Ministerio de Salud y en las Direcciones Seccionales de Salud. Este sistema presentaba problemas de cobertura y segmentación de la población, ineficiencia, falta de oportunidad y uso inadecuado de los recursos. En el SNS, las instituciones que funcionaban mediante el aseguramiento, es decir, el Instituto de Seguros Sociales y las Cajas de Previsión, llegaron a afiliar sólo el 21 \% de la población total del país. La reforma del sector salud hizo parte del proceso de reforma del Estado y el modelo adoptado se denominó “competencia regulada”, una combinación de elementos de mercado con elementos de regulación, dentro de los cuales podemos destacar la existencia de un paquete de beneficios conocido como Plan Obligatorio de Salud-POS, cuyo costo se establece por medio de la Unidad de Pago por Capitación-UPC. Hay dos tipos de POS: uno dirigido al régimen contributivo, POSc y otro para el régimen subsidiado, POSs.

El Plan Obligatorio de Salud debía haberse unificado para los dos regímenes (contributivo y subsidiado) en el año 2001. Esto no ocurrió y no sólo subsisten la baja cobertura, sino también un conjunto de problemas generados por la tensión entre la amplitud e integralidad del POSc y la falta de pertinencia y rigidez del paquete de servicios, frente a las necesidades regionales y la utilización real de servicios. A diferencia del Plan de Atención Básica-PAB que se ajusta periódicamente en cada entidad territorial, el POS ha sido construido desde el nivel nacional y los ajustes que se le han hecho se han basado principalmente en análisis económicos, más que en modelos probados que incluyan los perfiles epidemiológicos y la utilización de servicios como factores que se relacionan 
con el contenido, el costo y la sostenibilidad del paquete de servicios. En consecuencia, se requiere evaluar los perfiles epidemiológicos regionales y la utilización de los servicios, como parámetros para la adecuación del POS y la UPC.

\section{METODOLOGÍA}

Se realizó un estudio evaluativo de la problemática actual del SGSSS con base en entrevistas a expertos y revisión de fuentes secundarias, con el fin de establecer la cobertura, el gasto en salud, el financiamiento del sistema y los métodos de construcción del POS-UPC.

Se revisaron las metodologías utilizadas en el ámbito internacional para la conformación y la adecuación de paquetes de servicios, y se compararon con la experiencia de construcción del POS en Colombia.

De otra parte, se identificaron:

o Los factores que determinan la utilización de los servicios de salud en algunos países de Latinoamérica.

o Los parámetros de utilización de servicios en Colombia con base en la información de la Encuesta Nacional de Calidad de Vida del DANE de 1997, la Encuesta Nacional de Equidad en la Gestión Fiscal de la Contraloría General de la República de 1994 y la Encuesta Nacional de Hogares del DANE de 2000.

o Los modelos de regionalización del país y los perfiles epidemiológicos de cada región, con base en información de mortalidad de los Departamentos del año 2000, suministrada por el DANE, que se agregaron en sus respectivas regiones. Las causas de mortalidad se agruparon en grandes complejos epidemiológicos, definidos siguiendo la metodología de otro estudio (1).

A partir de los resultados obtenidos, se estableció la relación de los perfiles epidemiológicos con los contenidos del POS, de lo cual se presentan tan solo algunos comentarios que ilustran la utilidad de este tipo de análisis.

\section{RESULTADOS}

Problemáticas del SGSSS

Las problemáticas del SGSS identificadas se presentan de manera resumida en el cuadro a continuación. Dentro de este panorama complejo es importante destacar la ausencia de cobertura universal, el aumento del gasto en salud en la última década, la ausencia de unificación de los planes de beneficios y los problemas de equidad en el acceso a la atención. 


\begin{tabular}{|c|c|c|}
\hline Aspectos & Logros / beneficios & Insuficiencias / Problemas \\
\hline Afiliación & Incremento de aseguramiento & Nivel de afiliación insuficiente \\
\hline Gasto en salud & $\begin{array}{l}\text { Incremento sustancial: } 6 \text { a } 10 \% \\
\text { del PIB }\end{array}$ & $\begin{array}{l}\text { Poca eficacia, atesoramiento, costos } \\
\text { históricos }\end{array}$ \\
\hline Financiamiento & $\begin{array}{l}\text { Consolidación de cotizaciones } \\
\text { y recursos fiscales }\end{array}$ & $\begin{array}{l}\text { Se conservan las tres fuentes } \\
\text { sin reducir gasto de bolsillo }\end{array}$ \\
\hline Solidaridad & Vertical y horizontal & Parcial o insuficiente \\
\hline Pago & Contratos y formas múltiples & $\begin{array}{l}\text { Flujos inapropiados. Retardo, } \\
\text { comportamiento inadecuado de agentes }\end{array}$ \\
\hline Subsidios & $\begin{array}{l}\text { Creación del Régimen } \\
\text { Subsidiado }\end{array}$ & $\begin{array}{l}\text { POS incompleto, cobertura insuficiente, } \\
\text { esquema rígido }\end{array}$ \\
\hline Plan de beneficios & Paquete amplio & $\begin{array}{l}\text { Metodología de construcción y ajuste } \\
\text { indefinida. Falta de pertinencia. Falta de } \\
\text { unidad de planes. Segmentación }\end{array}$ \\
\hline Autonomía de prestadores & Se ha implantado & Débil en municipios pequeños \\
\hline Eficiencia & Eficiencia técnica parcial & No hay eficiencia asignativa \\
\hline $\begin{array}{l}\text { Viabilidad y sostenibilidad } \\
\text { de prestadores }\end{array}$ & $\begin{array}{l}\text { Esquema de rendición } \\
\text { de cuentas }\end{array}$ & $\begin{array}{l}\text { No hay régimen de transición. } \\
\text { Crisis hospitalaria generalizada }\end{array}$ \\
\hline Libertad de elección & Normatizada & No se ha avanzado \\
\hline Regulación y rectoría & $\begin{array}{l}\text { Funciones amplias apoyadas } \\
\text { en la normatividad }\end{array}$ & Rectoría casi inexistente \\
\hline $\begin{array}{l}\text { Descentralización } \\
\text { municipal }\end{array}$ & $\begin{array}{l}\text { Avances en autonomía y responsabili- } \\
\text { dad local }\end{array}$ & $\begin{array}{l}\text { Proceso de descentralización } \\
\text { incompleto }\end{array}$ \\
\hline $\begin{array}{l}\text { Eficacia } \\
\text { microeconomía }\end{array}$ & $\begin{array}{l}\text { Introducción de esquemas para } \\
\text { minimizar costos }\end{array}$ & \\
\hline Equidad. & $\begin{array}{l}\text { Mejoramiento de condiciones de afilia- } \\
\text { ción según ingreso. Mejoramiento en } \\
\text { distribución de la financiación y bajo } \\
\text { en distribución del gasto }\end{array}$ & No ha mejorado el acceso a los servicios \\
\hline
\end{tabular}

\section{Cobertura del SGSSS}

La incapacidad para lograr la cobertura universal que se planteó para el año 2000, es uno de los problemas más importantes del sistema. En la Tabla 1 se indica la cobertura alcanzada.

Tabla 1. Cobertura del SGSSS. Diciembre de 2001

\begin{tabular}{lll}
\hline Régimen & Número de afiliados & \% población total \\
\hline Contributivo & 13077930 & 30,3 \\
Subsidiado & 11062708 & 25,7 \\
No Afiliados & 19257453 & 44,0 \\
\hline TOTAL & 43035394 & 100,0 \\
\hline
\end{tabular}

Fuente: Conpes social 057, Distribución del sistema general de participaciones.

Vigencia 2002. Bogotá, 28 de Enero de 2002.

Los avances del Sistema General de Seguridad Social en Salud en cobertura, desde su implementación en el año 1993, no han sido suficientes y además ha sufrido un estancamiento en los años más recientes (2).

Esta deficiencia en los niveles de afiliación resulta de varios factores. El régimen contributivo que fue planeado para cubrir a cerca del 70 \% de la población colombiana, solamente llegó al 30,3 \% en el 2001; en cambio, el subsidiado que debería cubrir al 30 \% restante alcanzó un 25,7 \%. El desempleo en Colombia ha crecido significativamente a partir del año 98, a diferencia de los últimos años de la década del 80 y los primeros años de la del 90 cuando había disminuido de manera constante. 
Otro factor importante de esta insuficiente cobertura, ha sido la baja tasa de afiliación de la población trabajadora independiente al régimen contributivo, frente a los altos índices de informalidad en el trabajo que se observan en las principales ciudades.

Algunos estudios han explorado el tema del acceso a los servicios y la cobertura, confirmando el aumento de los niveles de afiliación entre la población más pobre por la entrada en vigencia del régimen subsidiado. La proporción de afiliados pasó de 3,1 \% a un 43,1 \% en el primer decil de ingresos, entre 1993 y 1997 (3). De acuerdo con la misma investigación, el número de consultas pasó de 598000 a 1,3 millones y el de hospitalizaciones aumentó un 50 \% en los mismos años.

El gasto en salud

A pesar de que los niveles de afiliación al SGSSS no han logrado alcanzar las metas propuestas en la Ley 100, es decir, la afiliación universal en el año 2001, el gasto en salud se ha incrementado de manera constante desde comienzos de la década anterior, pasando de un 7,2 \% del PIB en 1993 a 8,6 \% en el año1999, como se indica en la Tabla 2 (4).

Tabla 2. Gasto en salud como porcentaje del PIB

\begin{tabular}{|c|c|c|c|c|c|c|c|}
\hline Gasto & 1993 & 1994 & 1995 & 1996 & 1997 & 1998 & 1999 \\
\hline Gasto público & 3,5 & 3,3 & 4,3 & 5,2 & 5,3 & 5,1 & 5,5 \\
\hline Gasto privado & 3,6 & 3,0 & 3,2 & 3,6 & 3,9 & 4,2 & $4, .5$ \\
\hline Total & 7,2 & 6,3 & 7,4 & 8,8 & 9,3 & 9,3 & 8,6 \\
\hline
\end{tabular}

El incremento en el gasto en salud ha llegado, según algunos estudios, a representar el $10 \%$ del PIB, cerca de 15 billones de pesos del año 2000. El gasto privado, es decir, el gasto directo o de bolsillo que hacen las familias en salud también se ha incrementado, pasando de cerca de 3,8 billones de pesos en 1993 a casi 4,3 billones en 1999 (en pesos de 1999) (5); es decir, pasó de un 3,6 \% en 1993 a un 4,5 \% del PIB en 1999.

\section{Equidad en el SGSS}

Junto con los problemas de cobertura coexisten los de acceso a los servicios. Entre otros aspectos, el cobro de los copagos y las cuotas moderadoras se han convertido en obstáculo para la atención de los afiliados al sistema (6). Estas barreras económicas también aparecen al evaluar el acceso a los medicamentos, limitado por la pertenencia a uno u otro régimen de afiliación (7). En el régimen contributivo, el 32,7 \% de los beneficiarios y el 34,9 \% de los cotizantes no recibió los medicamentos. En el régimen subsidiado, el 43,9 \% no los recibió y en el grupo de los vinculados transitorios, el 44,3\% tampoco tuvo acceso a ellos. 
Con respecto a la satisfacción del usuario, la Encuesta Nacional de Calidad de Vida de 1997 reportó que 1628 de los encuestados manifestaron estar en alguno de los regímenes, mientras que 737 respondieron no tener ninguna afiliación; de los primeros el 75,3 \% asistió a consulta y el 24,7 \% no; de quienes asistieron a consulta, el 4,1 \% consideró que no lo examinaron, el 63,6 \% manifestó que se les ordenaron exámenes paraclínicos y al 78,2 \% le formularon medicamentos; en términos de oportunidad, los días transcurridos entre la solicitud de la consulta y su realización fue en el 42,7 \% de un día, 8,7 \% de dos días y $6 \%$ de tres días. Al calificar la atención, haciendo referencia a la amabilidad, el trato, las instalaciones y la información recibida el 36,5 \% la calificaron con 4 sobre 5 y el 34,9\% con 5; el 7,6 \% dieron calificaciones inferiores a 3.

Otro aspecto a considerar es la inadecuada distribución de los médicos, que están concentrados en las capitales (8). En la revisión de Ascofame se encontró que el país cuenta con 43166 médicos, lo que indica 10,4 médicos por 10 mil habitantes; de estos, 24 717, o sea 57,3 \% son médicos generales y de ellos el 26,4 \% se encuentran en Bogotá; de los médicos especialistas el 41 \% se encuentran en Bogotá, los demás se encuentran concentrados en Cali, Medellín y Barranquilla.

Frente a la incapacidad del sistema para lograr la cobertura universal se han presentado algunas alternativas de aseguramiento con subsidios parciales (9), de acuerdo con la capacidad económica de los usuarios, buscando la universalidad y la equidad en la contribución. Igualmente se ha encontrado que el sistema de identificación de los beneficiarios del régimen subsidiado-Sisben, presenta deficiencias tanto de exclusión de personas que deberían ingresar al régimen subsidiado y no logran hacerlo, como de inclusión errónea en el subsidio.

La construcción y la adecuación del POS y la UPC

La experiencia internacional acerca de la construcción de paquetes de servicios de salud pone el énfasis en el análisis de la morbilidad mediante los estudios de carga de la enfermedad (10), la estimación del costo - beneficio de las intervenciones en salud (11) y el ajuste del paquete de acuerdo con las necesidades de la población y la utilización de los servicios (12).

En Colombia, la formulación del POS, en el año 94, tuvo como parámetro principal el Decreto ley 1650 de 1977 que contenía las actividades del paquete de servicios para los afiliados al Instituto de Seguro Social. Adicionalmente el Ministerio de Salud (13), llevó a cabo una estimación de los años de vida saludable potencialmente perdidos-AVISA, que tienen en cuenta tanto la mortalidad como la carga de la enfermedad en términos de incapacidad. Para la construcción de los AVISA se clasificaron las enfermedades en 3 grupos: Transmisibles (grupo I), no transmisibles (grupo II) y ocasionadas por lesiones (grupo III). Se consideró que el grupo I representaba el 22,2 \% de las enfermedades, el grupo II el 39 \% y el grupo III el 38,8 \%. Generalmente, la carga de la enfermedad en el mundo difiere entre poblaciones rurales y urbanas, pero muy homogénea entre hombres y mujeres. Sin embargo, en Colombia el 65 \% de la carga de enfermedad corresponde a los hombres por el gran impacto que tienen las lesiones y los homicidios dentro de la carga total del país. Los hombres sufren 5 veces más lesiones que las mujeres. De la misma manera, no se presentan 
diferencias marcadas entre las áreas rurales y urbanas. En aquel momento se sugirió hacer una estimación de los AVISAS por Departamento, de manera que pudiesen establecerse las prioridades a escala regional y hacer más equitativa la asignación de recursos para salud.

La construcción de un paquete de servicios de salud tiene que ver con un proceso de priorización de las intervenciones más benéficas para atacar las causas más frecuentes e importantes de enfermedad y muerte en la población.

Esta priorización se hizo en nuestro país, en tres pasos:

o El estudio de cada enfermedad que represente una proporción significativa de la carga de enfermedad y los tratamientos asociados a ella.

o El estudio de las enfermedades en las cuales hay grandes avances preventivos y cuyas intervenciones son muy efectivas, como las enfermedades prevenibles mediante inmunización.

o La aproximación a un análisis de costo-efectividad de cada intervención y la selección de las más costo efectivas.

A pesar de lo anterior, no existen evidencias de los estudios de costo efectividad, ni de la construcción de conglomerados de actividades que permitieran un mejor abordaje de los problemas de salud del país. El contenido inicial del POS aparece en la Resolución 5261 de 1994 del Ministerio de Salud y dos modificaciones posteriores se reglamentaron en los Acuerdos 72 y 74 de 1997 del Consejo Nacional de Seguridad Social en Salud. En correspondencia con estas normas y con los demás documentos del Ministerio de Salud consultados, el paquete de servicios quedó compuesto por un amplio número de procedimientos, divididos en tres tipos:

- Promoción, prevención, diagnóstico y tratamiento ambulatorio en intervenciones muy costoefectivas que controlan problemas prioritarios en salud.

- Intervenciones que se entregan en hospitales y centros ambulatorios, no muy costo efectivas pero importantes para la salud general de la población.

- Algunas intervenciones poco costo efectivas, de alto costo para las familias, como la atención del cáncer, enfermedades cardiacas, transplantes y diálisis.

Las modificaciones de la UPC. La Unidad de Pago por Capitación es otro de los elementos creados por la Ley 100 de 1993 con el fin de establecer el costo del paquete de servicios para todo el país. Es otro de los mecanismos de regulación del sistema que evita la competencia por precios entre prestadores y aseguradores. En 1995 se le asignó un valor de \$ 121 000, en promedio. Posteriormente, en 1997 se definió una UPC para el régimen contributivo y otra para el subsidiado, de acuerdo con los diferentes contenidos de los dos paquetes de servicios. Esta diferencia se ha mantenido hasta el año 2003 y ha presentado además otras características tales como una estructura tarifaria diferencial por edad, sexo y porcentajes adicionales asignados para Departamentos con dificultades en el acceso a los servicios de salud. 
El análisis de los acuerdos que modifican el monto de la UPC a partir del año 1995, indican que la metodología inicial para calcular el aumento de la UPC en los años 96 y 97 consistió en aplicar el mismo porcentaje de incremento del salario mínimo en dichos años. A partir de 1998 y hasta el 2003 la metodología ha presentado dos aspectos básicos a señalar. El primero es la existencia, año tras año, de un déficit en la subcuenta de compensación del Fondo de Solidaridad y GarantíaFOSYGA que se ha empleado como parámetro para calcular el aumento. El segundo es la alusión a una serie de estudios técnicos adelantados por el Ministerio de Salud cada año, en cuanto al perfil epidemiológico, los riesgos cubiertos y los costos de prestación de los servicios, que se tienen en cuenta al calcular los aumentos de la UPC en el año respectivo (14).

La mortalidad por regiones en Colombia

Con base en la clasificación del Departamento Nacional de Planeación, las regiones del país se identificaron de la siguiente manera:

Occidente: Antioquia, Caldas, Cauca, Chocó, Nariño, Quindío, Risaralda y Valle del Cauca.

Centro Oriente: Cundinamarca, Boyacá, Tolima, Huila, Norte de Santander y Santander.

Costa Atlántica: Atlántico, Bolívar, Cesar, Córdoba, La Guajira, Magdalena, San Andrés y Sucre.

Orinoquia: Arauca, Casanare, Guainía, Guaviare, Meta, Vaupés y Vichada.

Amazónica: Amazonas, Putumayo y Caquetá.

Bogotá

Tabla 3. Mortalidad según causas y regiones. 2000. Tasas por 100 mil habitantes

\begin{tabular}{lrrrrr}
\hline \multirow{2}{*}{ Regiones } & \multicolumn{5}{c}{ Causas de mortalidad } \\
\cline { 2 - 6 } & \multicolumn{2}{c}{$\begin{array}{c}\text { Vasculares } \\
\text { accidentes }\end{array}$} & Tumores & Infecciosas & Perinatales \\
\hline Amazónica & 57 & 156 & 33 & 31 & 26 \\
Atlántica & 89 & 52 & 40 & 27 & 26 \\
Centro Oriente & 150 & 91 & 66 & 28 & 16 \\
Occidente & 131 & 144 & 79 & 31 & 16 \\
Orinoquía & 85 & 117 & 49 & 28 & 19 \\
Bogotá & 108 & 68 & 69 & 29 & 18 \\
\multicolumn{1}{c}{ Total } & 120 & 107 & 65 & & 19 \\
\hline
\end{tabular}

En la región Amazónica se destacan como causas de mortalidad los homicidios, las enfermedades isquémicas y vasculares, y las enfermedades respiratorias del período perinatal. Es de resaltar que aún hay mortalidad por enfermedades infecciosas respiratorias e intestinales para este año.

Atlántica: En esta región las enfermedades vasculares son la principal causa de mortalidad, seguida por las muertes violentas. Como en la región anterior las muertes provocadas por trastornos respiratorios en el período perinatal se encuentra dentro de las cinco primeras causas de mortalidad.

En la Región Centro-Oriente las causas más importantes corresponden a las enfermedades isquémicas y vasculares, los homicidios y los tumores malignos de Estómago que se ubican entre las primeras diez causas de mortalidad. 
Occidente: Esta región es la más afectada por los homicidios, seguidos por las enfermedades isquémicas y vasculares, y el cáncer gástrico, la cual se encuentra dentro las cinco primeras causas de mortalidad.

Orinoquía: En los últimos años los homicidios son la primera causa de mortalidad en esta región, seguidas por las enfermedades vasculares. También los trastornos respiratorios del período perinatal se encuentran dentro de las diez primeras causas de mortalidad.

En Bogotá las afecciones isquémicas y vasculares ocupan claramente el primer lugar, seguidas por los tumores y los homicidios.

Nacional: Los homicidios son la primera causa individual de mortalidad seguida por las enfermedades vasculares. Sin embargo, si se agregan las enfermedades isquémicas y vasculares estas pasan a ocupar el primer lugar.

En cuanto los sexos se evidencia que la mortalidad en el sexo masculino es más elevada por todas las causas y en todas las regiones, excepto con respecto a los tumores y la mortalidad por enfermedades vasculares. En el caso de los tumores, la mortalidad en el sexo femenino predomina en las regiones atlántica, occidental y en Bogotá, donde ocurre lo mismo para las enfermedades vasculares. El predominio de mortalidad por enfermedades crónicas en las mujeres se debe a que los hombres mueren relativamente jóvenes.

Es claro que las enfermedades vasculares se encuentran como una de las principales causas de mortalidad en todas las regiones; en las regiones Centro Oriente y Occidente es la principal causa de mortalidad. Por edad el grupo principalmente afectado es el de mujeres de 45 a 64 años. Por lo anterior, en estas regiones el POS debería incluir las medidas de detección temprana, así como las valoraciones específicas de laboratorio y examen anual, apropiadas para afrontar este tipo de problemas, en promoción, prevención y atención.

En segundo lugar, encontramos las muertes violentas y accidentes; sin embargo, al desagregar las causas es necesario resaltar que en el ámbito nacional los homicidios constituyen la primera causa individual de muerte, y la población masculina es la más vulnerable con un predominio de edad entre los 15 a 44 años. Las regiones Amazónica y Occidental son las más afectadas por este fenómeno, con las mismas características por edad y sexo que las del país. En esta dimensión son innumerables los aspectos que deben ser considerados en función del perfeccionamiento del POS, ya que estos fenómenos tienen múltiples causas y manifestaciones.

La mortalidad por tumores predomina en la zona Occidental y en Bogotá. El grupo de 45 a 64 años y las mujeres son la población principalmente afectada por estas patologías. Al revisar las causas desagregadas el Cáncer Gástrico cobra importancia debido a que registra las tasas de mortalidad más elevadas, lo que representa la necesidad de la inclusión de actividades para la 
detección temprana en las zonas de alto riesgo así como las que existen para el cáncer de cervix y seno a escala nacional.

En cuanto a las enfermedades infecciosas se encuentra una mortalidad similar en las tasas por 100 mil habitantes entre las zonas Amazónica, Orinoquia y Occidental, siendo más frecuente en los hombres y afectando a los grupos etáreos de menores de un año y mayores de 65 . En las causas desagregadas las infecciones respiratorias agudas y las enfermedades infecciosas intestinales son las de mayor importancia en la zona Amazónica, y las infecciones respiratorias en la Occidental. El fortalecimiento de la atención del primer nivel puede contribuir a la disminución de muertes por estas causas previsibles. En el mismo sentido, sería necesario considerar los contenidos específicos del POS que deben responder a problemáticas como las enfermedades tropicales, en programas como el control de vectores, la detección temprana y control de la enfermedad con el fin de reducir la mortalidad por dichas causas.

La diversidad regional tiene su contrario, o sea patologías comunes a todas las regiones o a gran parte de ellas. Este aspecto toca un amplio contenido del POS que es necesario en todo el país y debe ser fortalecido. Quizás el mejor ejemplo, más no el único, es la problemática conectada con la mortalidad por violencia, homicidios y accidentes, las cuales requieren un complejo y extenso repertorio de acciones y actividades en el POS, que no son consideradas actualmente.

\section{La utilización de los servicios de salud}

La Encuesta Nacional de Calidad de Vida de 1997, realizada por el DANE tomó 9121 hogares con un total de 38516 personas encuestadas. En el capítulo dedicado a salud incluyó preguntas sobre afiliación, morbilidad, utilización y pago de los servicios. Esta información se estratificó por sexo (masculino y femenino), rangos de edad en años (0-14, 15-49 y 50 o más), lugar de residencia (rural o urbano) y quintil de gasto ( 1 a 5 ) siendo 1 el quintil de gasto más bajo y el 5 más alto. En la Tabla 4 se presentan los resultados de la variable "problema de salud en los últimos 30 días". Los resultados se presentan por quintil de gasto, sexo y lugar de residencia. El $17 \%$ de los hombres, y el 23,1 \% de las mujeres de la zona urbana declararon haber tenido algún problema de salud. El promedio para zona urbana fue de 20,6 \%. Para la zona rural los porcentajes fueron muy similares, $17,8 \%, 23$ y $20,2 \%$ respectivamente.

A continuación se indica la proporción de quienes teniendo problemas de salud recibieron atención, entendiendo por esta la prestada únicamente por un profesional, institución de salud (Hospital, clínica, centro o puesto de salud), enfermera o promotor (Tabla 5). Los resultados muestran una mayor utilización de servicios de salud a medida que se incrementa la edad, de 15 años en adelante y a medida que se incrementa el gasto, del quintil 2 en adelante. 
Tabla 4. Problemas de salud (\%) en los últimos treinta días según área de residencia y sexo por quintil de gasto, Colombia 1997

\begin{tabular}{ccccccc}
$\begin{array}{c}\text { Quintil de } \\
\text { gasto salud }\end{array}$ & $\begin{array}{c}\text { Hombres } \\
\text { urbano }\end{array}$ & $\begin{array}{c}\text { Mujeres } \\
\text { urbano }\end{array}$ & $\begin{array}{c}\text { Total } \\
\text { urbano }\end{array}$ & $\begin{array}{c}\text { Hombres } \\
\text { rural }\end{array}$ & $\begin{array}{c}\text { Mujeres } \\
\text { rural }\end{array}$ & $\begin{array}{c}\text { Total } \\
\text { rural }\end{array}$ \\
\hline 1 & 16,8 & 18,0 & 17,4 & 12,5 & 15,6 & 14,0 \\
2 & 16,5 & 21,9 & 19,3 & 16,5 & 20,5 & 18,3 \\
3 & 17,4 & 23,4 & 20,6 & 16,4 & 22,7 & 19,4 \\
4 & 17,7 & 26,8 & 22,5 & 21,5 & 24,9 & 23,1 \\
5 & 21,2 & 25,2 & 23,4 & 21,9 & 31,2 & 26,0 \\
\hline Promedio & 17,9 & 23,1 & 20,6 & 17,8 & 23,0 & 20,2
\end{tabular}

Fuente: DANE. Encuesta Nacional de Calidad de Vida 1997.

Tabla 5. Atención en salud (\%) por problemas en los últimos 30 días según grupos de edad, por quintil de gasto Ambos sexos. Colombia 1997

\begin{tabular}{|c|c|c|c|c|}
\hline Quintil de gasto & 0-14 años & $\begin{array}{c}\text { 15-49 } \\
\text { años }\end{array}$ & $\begin{array}{c}50 \text { y más } \\
\text { años }\end{array}$ & Total \\
\hline 1 & 53,9 & 66,1 & 64,9 & 61,7 \\
\hline 2 & 72,0 & 69,8 & 78,8 & 72,5 \\
\hline 3 & 74,3 & 82,0 & 83,0 & 80,0 \\
\hline 4 & 86,6 & 83,2 & 91,1 & 86,0 \\
\hline 5 & 89,0 & 84,7 & 91,5 & 87,3 \\
\hline Promedio & 75,1 & 77,2 & 81,8 & 77,5 \\
\hline
\end{tabular}

Fuente: DANE. Encuesta Nacional de Calidad de Vida. Colombia 1997

Los datos anteriores también sugieren que el 22,5 \% de las personas no acuden a un servicio de salud para solucionar sus problemas sino que buscan otras alternativas.

Por área de residencia y sexo se encuentran varios elementos. La mayor utilización de servicios es de las mujeres que residen en zonas urbanas e igualmente en el quintil de gasto más alto. La menor es de los hombres de la zona rural del primer quintil de gasto ya que solamente el 50,7 \% de los que se sintieron enfermos acudieron al servicio de salud (Tabla 6).

De otra parte, se observa un mayor promedio de consulta en la zona urbana en todos los quintiles de gasto, así como en las mujeres frente a los hombres. El promedio rural fue casi $10 \%$ menor que el urbano.

En todas las encuestas se observan varios hechos:

o La mayor utilización de servicios por parte de las mujeres frente a los hombres en las dos áreas de residencia y en todos los quintiles de gasto.

o La utilización en el área urbana es mayor que en el área rural en los dos sexos y en todos los quintiles de gasto.

o La utilización de servicios se incrementa al ascender los ingresos, tanto en el área rural como urbana e igualmente por sexo. 
Tabla 6. Atención en salud (\%) por problemas en los últimos treinta días según área de residencia y sexo por quintil de gasto. Colombia 1997

\begin{tabular}{ccccccc}
\hline $\begin{array}{c}\text { Quintil de } \\
\text { gasto }\end{array}$ & $\begin{array}{c}\text { Hombres } \\
\text { Urbano }\end{array}$ & $\begin{array}{c}\text { Mujeres } \\
\text { Urbano }\end{array}$ & $\begin{array}{c}\text { Total } \\
\text { Urbano }\end{array}$ & $\begin{array}{c}\text { Hombres } \\
\text { Rural }\end{array}$ & $\begin{array}{c}\text { Mujeres } \\
\text { Rural }\end{array}$ & $\begin{array}{c}\text { Total } \\
\text { Rural }\end{array}$ \\
\hline 1 & 59,4 & 73,6 & 67,0 & 50,7 & 63,6 & 57,7 \\
2 & 72,9 & 80,1 & 77,1 & 62,8 & 65,7 & 64,3 \\
3 & 82,2 & 83,0 & 82,7 & 62,9 & 77,2 & 70,8 \\
4 & 86,8 & 87,8 & 87,4 & 75,5 & 78,2 & 76,8 \\
5 & 85,5 & 88,9 & 87,5 & 83,7 & 84,1 & 83,9 \\
\hline Promedio & 77,4 & 82,7 & 80,3 & 67,1 & 73,8 & 70,7 \\
\hline
\end{tabular}

Fuente: DANE. Encuesta Nacional de Calidad de Vida. Colombia 1997.

La utilización de servicios de promoción y prevención o consulta preventiva fue evaluada únicamente en la Encuesta Nacional de Calidad de Vida de 1997. La pregunta “¿Sin estar enfermo y por prevención consulta al médico o al odontólogo por lo menos una vez al año?” dio resultados similares a los de la utilización por problemas de salud; es decir, la consulta preventiva fue mayor en las mujeres que en los hombres en todos los niveles de gasto y fue mayor también en la zona urbana que en la rural, con una diferencia de 10,6 \% de la primera sobre la segunda.

\section{¿Cómo adecuar el POS-UPC?}

El ajuste del POS-UPC es uno de los medios, entre otros, para contribuir a la ampliación de la afiliación al SGSSS, en busca de la universalidad. Este ajuste no implica eliminar servicios del POS ni recortar los derechos de los ciudadanos a recibir estos servicios. En estas condiciones para acoplarse a la utilización real o estimada de los servicios de atención y a la especificidad epidemiológica regional, se requieren por lo menos dos aspectos:

- Un POS modular flexible, con grupos de servicios que responden a las necesidades presentes en todo el territorio nacional, a las específicas regionales y a las patologías catastróficas, según su utilización diferencial.

- Los recursos que quedan disponibles al ajustar la UPC según el esquema anterior, pasan a un fondo de estabilización y ampliación de cobertura cuyas funciones principales serían dos: a. Proteger el acceso integral al POS si la utilización de los servicios o las diferencias epidemiológicas regionales operan de una manera diferente a la esperada en un momento dado; b. Proveer fondos para nuevas afiliaciones, por medio de un plan de liberación progresiva de los fondos disponibles.

Adicionalmente se requiere precisar cómo están operando actualmente los costos de atención de las IPS, los costos de administración de las EPS y la utilidad de las mismas. Esto último debido a la insuficiente información sobre los márgenes de utilidades que obtienen las EPS y la carencia de esquemas uniformes para estimarlas. 


\section{DISCUSIÓN}

El presente estudio se ha ocupado de evaluar los perfiles epidemiológicos regionales y la utilización de los servicios de salud en Colombia, con el fin de determinar su utilidad como parámetros de adecuación del plan de beneficios del SGSSS. Este tipo de ejercicios tendría un mayor alcance si pudiésemos contar con información actualizada sobre la morbilidad y los riesgos en el país y las regiones.

En el país, la construcción del POS-UPC ha sido poco clara e insuficiente, y no se ha documentado ni se han analizado los parámetros apropiados para su adecuación progresiva, a pesar de que varias normas insisten en ello.

Si bien creemos que se debe conservar y proteger la integralidad del POS, su estructura única nacional, lo torna rígido y con frecuencia genera una aplicación no pertinente, poco oportuna y sin la calidad adecuada en la prestación de servicios. La adecuación del POS y la UPC con base en la utilización real de los servicios y los perfiles epidemiológicos, conservando su amplitud y alcance, puede contribuir de manera significativa a superar estos problemas de oportunidad, pertinencia y calidad, sirviendo al tiempo para avanzar en el propósito de universalidad.

La adecuación del POS no debe enmarcarse en una interpretación simplista de la universalidad. Esta corresponde a un proceso complejo que está determinado por factores de orden estructural, de tipo situacional, y por las políticas que inciden sobre la afiliación de manera directa o indirecta. Cabe destacar:

- Las variables y tendencias de orden estructural: modelo de desarrollo, situación de la economía y empleo.

- Los recursos fiscales o recursos del Estado dedicados a la afiliación de la población pobre.

- La superación de la evasión y el control de la elusión.

- Posibles políticas y métodos para incorporar a la población ubicada en la economía informal al SGSSS: refinamiento del Sisben, subsidios parciales, incorporación automática y estratificada, entre otras

\section{REFERENCIAS}

1. Ministerio de Salud. La Salud en Colombia. Diez años de información. Santa fe de Bogotá; 1994.

2. Universidad de Antioquia. Centro de investigaciones económicas. Cobertura del seguro de salud en Colombia. Junio 2001; Año 1 No. 1.

3. Céspedes J, Jaramillo I. Efectos de la reforma de la seguridad social en salud en Colombia sobre la equidad en el acceso y la utilización de servicios de salud. Revista salud pública 2000; 2 (2): 145-165. 
4 Salud Colombia [Internet]. Departamento Nacional de Planeación. Cuentas Nacionales. Disponible en: http://www.saludcolombia.com Edición 59, Junio 2001. Consultado Julio de 2003.

5. Martínez F, Robayo G, Valencia O. ¿'Por qué no se logra la cobertura universal de la seguridad social en salud? Fundación para la investigación y desarrollo de la salud y la seguridad social. Bogotá; 2003.

6. Giraldo J. Primera encuesta nacional de calidad en salud percibida por los usuarios. Defensoría del pueblo, Bogotá; 1999-2000.

7. Mejía S, Vélez A, Buriticá O, Arango M, Del Río J. Evaluación de la política en términos de accesibilidad y uso racional en Colombia. Manizales; 1999.

8. ASCOFAME. Recurso humano en medicina. Bogotá; 2000.

9. Arévalo D, Martínez F, Rodríguez O. ¿Ha mejorado el acceso en salud?. Evaluación de los procesos del régimen subsidiado. Universidad nacional de Colombia. Centro de investigaciones para el desarrollo. Ministerio de salud; Noviembre de 2002.

10. Bobadilla J, Cowley P, Musgrove P, et al. Diseño, contenido y financiamiento de un paquete nacional de servicios de salud esenciales. Boletín Oficina Sanitaria Panamericana 1995;118 (2).

11. Bobadilla J. Searching for essential health services in low- and middle- income countries. Inter American Development Bank. Washington; June 1998.

12. Programa FLAGSHIP. Focalización y paquetes de beneficios. Capítulo 2: definición de un paquete básico de servicios de salud. Santiago de Chile; 2000.

13. Ministerio de Salud. La reforma de la seguridad social en salud. Antecedentes y resultados. Santa fe de Bogotá; 1994.

14. Consejo Nacional de Seguridad Social en Salud. Acuerdos Nº 011 de 1995, 024 de 1996, 050 de 1997, 084 de 1998, 119 de 1999, 159 de 2000, 186 de 2001, 218 de 2002 y 240 de 2003. 
